\title{
Progress towards Elimination of HIV Mother-to-Child Transmission in the Dominican Republic from 1999 to 2011
}

\author{
Osvaldo Lorenzo, ${ }^{1}$ Consuelo M. Beck-Saguée, ${ }^{2,3}$ Claudia Bautista-Soriano, ${ }^{4,5}$ Mina Halpern, ${ }^{6}$ \\ José Roman-Poueriet, ${ }^{6,7}$ Nora Henderson, ${ }^{3,8}$ Eddy Perez-Then, ${ }^{9}$ Rosa Abreu-Perez, ${ }^{10}$ \\ Solange Soto, ${ }^{10}$ Luis Martínez, ${ }^{11}$ Sarah Rives-Gray, ${ }^{12}$ Bienvenido Veras, ${ }^{13}$ \\ Maureen Connolly, ${ }^{14}$ Greer Brittany Callender, ${ }^{6}$ and Stephen W. Nicholas ${ }^{15,16}$
}

\author{
${ }^{1}$ Dirección General de Control de las Infecciones de Transmisión Sexual y SIDA (DIGECITSS), Ministerio de Salud Pública (MSP), \\ Santo Domingo, Dominican Republic \\ ${ }^{2}$ Robert Stempel College of Public Health and Social Work, Florida International University, Miami, \\ FL 33199, USA \\ ${ }^{3}$ Clinton Foundation Health Access Initiative, Santo Domingo, Dominican Republic \\ ${ }^{4}$ United Nations Children's Fund (UNICEF), Santo Domingo, Dominican Republic \\ ${ }^{5}$ Consejo Presidencial del SIDA (COPRESIDA), Santo Domingo, Dominican Republic \\ ${ }^{6}$ Clinica de Familia La Romana, La Romana, Dominican Republic \\ ${ }^{7}$ USAID/PEPFAR-Family Health International (FHI), Santo Domingo, Dominican Republic \\ ${ }^{8}$ School of Medicine, Department of Medicine, New York University (NYU), New York, NY 10012, USA \\ ${ }^{9}$ Centro Nacional de Investigaciones en Salud Materno Infantil Dr. Hugo Mendoza (CENISMI), \\ Santo Domingo, Dominican Republic \\ ${ }^{10}$ Hospital Infantil Robert Reid Cabral, Santo Domingo, Dominican Republic \\ ${ }^{11}$ Hospital Materno Infantil San Lorenzo de los Mina, Santo Domingo, Dominican Republic \\ ${ }^{12}$ Hospital Francisco Gonzalvo, La Romana, Dominican Republic \\ ${ }^{13}$ Hospital Regional Universitario José María Cabral y Báez, Santiago de los Caballeros, Dominican Republic \\ ${ }^{14}$ Children's Hospital of Philadelphia, Residency Program in Pediatrics, Philadelphia, PA 19104, USA \\ ${ }^{15}$ Mailman School of Public Health, Columbia University, New York City, NY 10032, USA \\ ${ }^{16}$ Columbia University International Family AIDS Program, La Romana, Dominican Republic
}

Correspondence should be addressed to Consuelo M. Beck-Sagué, becksague@gmail.com

Received 6 July 2012; Accepted 16 September 2012

Academic Editor: Carmen Zorrilla

Copyright (C) 2012 Osvaldo Lorenzo et al. This is an open access article distributed under the Creative Commons Attribution License, which permits unrestricted use, distribution, and reproduction in any medium, provided the original work is properly cited.

In 1999, prevention of mother-to-child transmission (pMTCT) using antiretrovirals was introduced in the Dominican Republic (DR). Highly active antiretroviral therapy (HAART) was introduced for immunosuppressed persons in 2004 and for pMTCT in 2008. To assess progress towards MTCT elimination, data from requisitions for HIV nucleic acid amplification tests for diagnosis of HIV infection in perinatally exposed infants born in the DR from 1999 to 2011 were analyzed. The MTCT rate was $142 / 1,274$ (11.1\%) in 1999-2008 and 12/302 (4.0\%) in 2009-2011 $(P<.001)$, with a rate of 154/1,576 (9.8\%) for both periods combined. This decline was associated with significant increases in the proportions of women who received prenatal HAART (from $12.3 \%$ to $67.9 \%$ ) and infants who received exclusive formula feeding (from $76.3 \%$ to $86.1 \%$ ) and declines in proportions of women who received no prenatal antiretrovirals (from $31.9 \%$ to $12.2 \%$ ) or received only single-dose nevirapine (from 39.5\% to $19.5 \%$ ). In 2007 , over $95 \%$ of DR pregnant women received prenatal care, HIV testing, and professionally attended delivery. However, only $58 \%$ of women in underserved sugarcane plantation communities (2007) and 76\% in HIV sentinel surveillance hospitals (2003-2005) received their HIV test results. HIV-MTCT elimination is feasible but persistent lack of access to critical pMTCT measures must be addressed. 


\section{Introduction}

HIV mother-to-child transmission (MTCT) continues to threaten child survival worldwide. Over two million children are infected with HIV, the vast majority infected by MTCT in low- and middle-income (LMI) countries $[1,2]$. The persistence of HIV MTCT underscores the importance of increasing access to effective prevention of MTCT (pMTCT) strategies. Virtual elimination of HIV MTCT has been achieved in most industrialized countries, with declines of over $80 \%-90 \%$ in the number of cases of perinatally acquired HIV infection, and MTCT rates of under 2\%-3\% [3-5]. The use of highly active antiretroviral therapy ([HAART], defined as a regimen of three or more antiretrovirals from two or more classes), which is essential for elimination of MTCT and contributes to maternal health, has been shown to be cost effective compared to single-dose nevirapine in several LMI countries, including the Dominican Republic (DR) [68], but few data exist on the scalability of these measures or the current obstacles to eliminating MTCT in these countries [9-13].

The DR is home to $25 \%$ of persons living with HIV in the Caribbean, the second highest HIV-prevalence region in the world $[1,9,10]$. To assess progress towards MTCT elimination in this MI country, we analyzed data recorded at the time of collection of samples for virologic testing performed to determine whether perinatally exposed infants and young children had acquired HIV infection.

\section{Methods}

2.1. Population. Guardians of infants and children known or suspected of being perinatally HIV-exposed were asked if they would consent to diagnostic virologic testing of their children as part of pediatric care. The population tested included infants and children whose mothers were diagnosed with HIV infection before or during pregnancy, at delivery, and up to 25 months after the child's birth. Since HIV infection is a frequent cause of death among Caribbean women of reproductive age [10], HIV antibody testing was performed in orphaned and abandoned minors if maternal serostatus was unavailable. If the patient was seropositive, his/her guardian was offered virologic testing. A case of HIV infection acquired by MTCT was defined as an infant with a positive HIV nucleic acid amplification test [11]. Infants who had negative HIV nucleic acid amplification tests and had no symptoms of HIV infection were considered uninfected, but were followed until their HIV antibody tests were negative.

2.2. Prenatal and Infant pMTCT Practices. From 1999-2004, in La Romana province, in the region that formerly had the highest HIV prevalence in 2002 [12], nongovernmental organizations provided prenatal, perinatal and neonatal multidose zidovudine-containing regimens, with elective cesarean delivery and exclusive formula feeding, to HIVinfected mothers and their infants $[6,13]$. During this time period, all data for this paper were collected from La Romana (Figure 1). Outside of La Romana, most HIVinfected women who were diagnosed during pregnancy

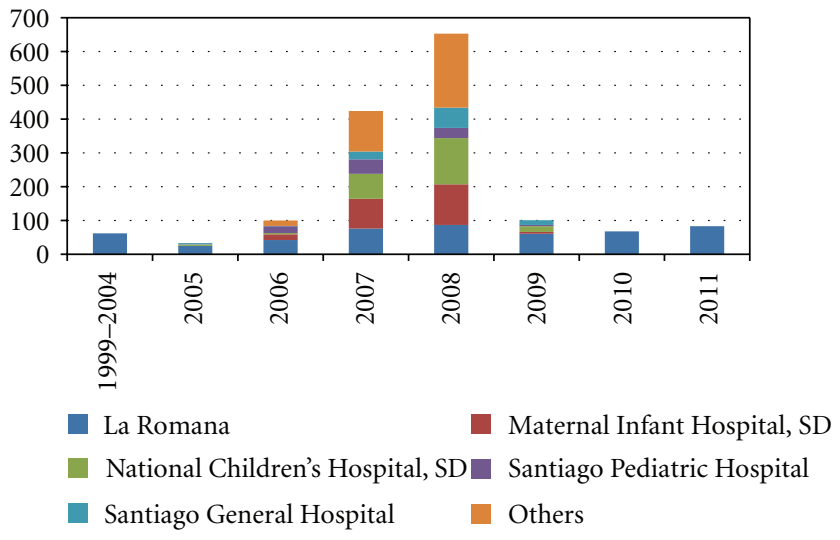

Figure 1: Perinatally HIV-Exposed Infants, by 34 Reporting centers, Dominican Republic, 1999-2011 "La Romana": nongovernmental organization (NGO) located in La Romana, in southeastern Dominican Republic, provides prenatal care to $81-101$ pregnant women living with HIV annually, approximately $30 \%$ of Haitian origin, and 20\% from bateyes (underserved sugarcane plantation communities); virtually all $(>97 \%)$ are low-income informally employed or unemployed $[6,13]$. The NGO provides comprehensive care to approximately 1,300 persons living with HIV, of whom 130 are children. La Romana is a major sugar-producing area, with $100 \mathrm{~s}$ of bateyes for workers. It is also a major tourist attraction. "Maternal Infant Hospital, SD": public maternity hospital, located in Santo Domingo (SD) East, in the southern coast of the Dominican Republic [14], reports over 10,800 live births, at least 800 to women living with HIV. Most of the clients are low income, and over $20 \%$ may be of Haitian origin. The hospital provides comprehensive care to 90-101 children living with HIV. "National Children's Hospital, SD": Public children's hospital located in SD, DR capital city, in the southern coast of the DR, reports $>6,000$ admissions per year, and evaluates 150-200 perinatally exposed infants per year. Most of the clients are low-income, and at least $20 \%$ are referred to tertiary services in the Children's Hospital from other areas. The hospital provides comprehensive care to over 400 children living with HIV. "General Hospital, Santiago": public general hospital located in the Northern city of Santiago de los Caballeros, reports approximately 23,000 admissions per year, of which 10,000 are obstetric, $90-120$ of which are among women living with HIV. Although it is located in one of the more economically prosperous areas of the DR, it serves a diverse population and many clients are from impoverished border areas. The hospital provides comprehensive care to over 1,300 adults living with HIV, and prenatal care services to over 100 women living with HIV annually. "Pediatric Hospital, Santiago": public children's hospital located in the Northern city of Santiago de los Caballeros, reports over 3,000 admissions per year, and evaluates 50-60 perinatally exposed children per year. The hospital provides comprehensive care to approximately 140 children living with HIV. The other 29 centers that are represented in this population include two community based, three faith-based, and two other NGOs, and 22 public hospitals in the eastern, border, central, and southwestern provinces, including Elias Pina, El Seibo, and other low-healthcareaccess areas $[12,15,16]$.

were offered single-dose nevirapine at delivery, and exclusive formula feeding for their infants, according to national guidelines [17]. From 2004-2008, some women diagnosed with HIV infection before or during pregnancy started or continued HAART if they needed it for immunodeficiency; 
others received single-dose nevirapine or a zidovudinecontaining multidose regimen [13]. In 2008, the DR ministry of health issued guidelines recommending maternal HAART for pMTCT, whether or not the mother had evidence of immunodeficiency (Protocol for use of combined antiretroviral therapy in pregnant women living with HIV/AIDS, Ministry of Health, Santo Domingo, DR, October 2008). These guidelines called for initiating zidovudine and lamivudine combined with nevirapine if the woman's CD4+ Tlymphocyte count was less than 250 cells per $\mathrm{mm}^{3}$ [18] or ritonavir-boosted lopinavir if the woman's $\mathrm{CD} 4+\mathrm{T}$ lymphocyte count was higher. HAART users with a documented quantitative HIV RNA concentration ("viral load") less than 1,000 viral particles $/ \mathrm{ml}^{3}$ were advised to deliver vaginally; non-HAART users and those with unknown or higher viral loads were encouraged to undergo elective cesarean delivery.

2.3. Laboratory Testing. Plasma specimens were obtained at age one to 25 months from known perinatally exposed and/or HIV-antibody-positive infants born from 1999-2005 for DNA polymerase chain reaction (PCR) qualitative or quantitative HIV-1 "viral load" testing. If viral nucleic acid was detected, testing was repeated. If the result was negative and the sample had been obtained prior to six months of age, the test was repeated at age of six months. From 20062011, dried blood spots (DBS) obtained from perinatally exposed and/or HIV-antibody-positive infants and children aged one to 25 months were collected according to previously published procedures and tested for viral nucleic acid [19].

2.4. Data Abstraction, Processing and Analysis. Information regarding maternal HIV diagnosis, prenatal antiretroviral treatment, delivery, and infant antiretroviral treatment and nutrition was obtained from maternal and infant medical records from 1999 to 2005. Physicians and nurses providing care for perinatally HIV-exposed infants queried the parents or guardians about this information 2006-2011 and documented their responses on laboratory requisition forms for HIV PCR testing on DBSs when the parent or guardian consented to the infant's HIV PCR test. No attempts were made to verify the data obtained, beyond reviewing maternal or infant records, if available. When guardians did not have the information, and it was not found in requisitions or records, values were coded as missing.

Data were entered into Excel databases, (one in La Romana, and the other for the rest of the sites) and updated with the DBS HIV test results when they were received. In 2012, the Excel databases were converted to Epi Info v3.5.1 for Windows (Centers for Disease Control and Prevention, 2008) files, the variables were harmonized, and the databases were merged and analyzed [20].

HIV MTCT rates per 100 infants and children were calculated for maternal and infant antiretroviral regimen, delivery and infant nutrition characteristics, year and period (pre- and post-HAART for pMTCT guidelines) of birth, and by the various pMTCT strategies. Chi-square or Fisher's exact two-tailed tests were used to evaluate categorical variables for statistical significance. The Kruskal-Wallis test for two groups was used for continuous variables. Stratified analyses were performed to control for confounding.

2.5. Prenatal and Infant Feeding Practices. Proportions of women who attended prenatal care, had hospital deliveries and breastfed were obtained from 2007 Demographic and Health Survey (DHS) data [12]. Data on HIV in bateyes (underserved sugarcane plantation communities) were obtained from a DHS 2007 component [15]. Updates on pMTCT strategies in 2003-2005, 2009, and HIV prevalence estimates, projections, and national prevention objectives in 2009 were reviewed $[14,16,21]$.

\section{Results}

3.1. Population. Data from 1,576 infants and children born from December 1, 1999 to December 31, 2011 were analyzed. These included data collected in 34 centers from 1,274 infants born from 1999-2008, and in 18 centers from 302 infants born from January 1, 2009 to December 31, 2011 (Figure 1). Age at specimen collection ranged from 20 days to 25 months $($ median $=3.0$ months; mean $=4.7 ;$ s.d. $=3.9 ;$ mode $=2.0$ months); $1,541(97.8 \%)$ samples were collected before the child was 15 months old.

\subsection{Prenatal, Delivery, and Postpartum pMTCT Practices.} From 1999 to 2008, HIV infection was diagnosed during the pregnancy in $48.4 \%$ mothers, prior to the pregnancy in $21.7 \%$, and during labor or within 72 hours of birth in $5.7 \%$ and $5.2 \%$, respectively (Table 1). Maternal HIV infection was diagnosed after 72 hours and during the child's infancy or early childhood in $12.6 \%$. In $6.3 \%$, testing was performed and maternal HIV infection was diagnosed at other or unknown times. After publication of the national guidelines recommending HAART for pMTCT (2009-2011), the proportion diagnosed during labor and delivery rose and the proportions diagnosed within 72 hours of birth and at other or unknown times declined significantly. Otherwise, the distribution did not change significantly from the 1999 to 2008 period.

Maternal antiretroviral use during pregnancy, labor, or delivery rose significantly (Table 1 ). Women in the earlier period were significantly more likely to receive a single-dose nevirapine regimen than women who delivered from 2009 to 2011. Conversely, women who delivered from 2009 to 2011 were significantly more likely to receive HAART than in 1999 to 2008. All 157 women who received HAART before 2009 had CD4+ T-lymphocyte counts less than 250 per $\mathrm{mm}^{3}$. The majority (57.6\%) of women who delivered in 2009-2011 received HAART, and most of these HAART recipients had CD4 counts higher than 250 and received ritonavir-boosted lopinavir. Duration of nevirapine-containing HAART at delivery ( median $=286$ days) was longer than the duration of lopinavir-containing HAART ( median $=104$ days; $P<.001$ ); mothers who used nevirapine-containing HAART were more likely to have started it before pregnancy than those who used lopinavir-containing HAART. Use of other multidose 
TABLE 1: Characteristics of maternal HIV diagnosis, maternal and infant antiretroviral use, mode of delivery and infant feeding, Dominican Republic, 1999-2008, and 2009-2011.

\begin{tabular}{|c|c|c|}
\hline \multirow{3}{*}{ Diagnosis and management } & $1999-2008$ & $2009-2011$ \\
\hline & No. (\%) & No. (\%) \\
\hline & $N=1,274$ & $N=302$ \\
\hline \multicolumn{3}{|l|}{ Time of HIV diagnosis } \\
\hline Before pregnancy & $277(21.7)$ & $77(25.5)$ \\
\hline During pregnancy & $617(48.4)$ & $143(47.4)$ \\
\hline Labor/delivery & $73(5.7)$ & $38(12.6)^{*}$ \\
\hline$\leq 72$ hours post-partum & $66(5.2)$ & $5(1.7)^{*}$ \\
\hline After 72 hours & $160(12.6)$ & $38(12.6)$ \\
\hline Unknown & $81(6.3)$ & $1(0.3)^{*}$ \\
\hline \multicolumn{3}{|l|}{ Maternal antiretroviral use } \\
\hline Any & $868(68.1)$ & $265(87.7)^{*}$ \\
\hline HAART, either & $157(12.3)$ & $205(67.9)^{*}$ \\
\hline $\mathrm{AZT}+3 \mathrm{TC}+\mathrm{NVP}^{1}$ & $157(12.3)$ & $59(19.5)$ \\
\hline AZT-3TC-Lop² & - & $146(48.3)^{*}$ \\
\hline Multidose AZT & $19(1.5)$ & 0 \\
\hline Multidose AZT + 3TC & $61(4.8)$ & - \\
\hline Single dose $\mathrm{NVP}^{3}$ & $503(39.5)$ & $60(19.5)^{*}$ \\
\hline Received no ARVs & $406(31.9)$ & $37(12.2)^{*}$ \\
\hline Unknown & $121(9.5)$ & $0^{*}$ \\
\hline \multicolumn{3}{|l|}{ Type of delivery } \\
\hline Vaginal & $285(22.4)$ & $81(26.8)$ \\
\hline All Caesareans & $882(69.2)$ & $199(65.9)$ \\
\hline Elective caesarian & $882(69.2)$ & $152(50.3)$ \\
\hline Emergency caesarean & - & $47(15.6)$ \\
\hline Unknown & $107(8.4)$ & $22(7.3)$ \\
\hline \multicolumn{3}{|l|}{ Infant nutrition } \\
\hline Formula only & $972(76.3)$ & $260(86.1)^{*}$ \\
\hline Breast feeding only & $67(5.3)$ & $4(1.3)^{*}$ \\
\hline Both & $43(3.4)$ & $13(4.2)$ \\
\hline Unknown & $192(15.1)$ & $25(8.3)^{*}$ \\
\hline \multicolumn{3}{|l|}{ Infant antiretrovirals (ARVs) } \\
\hline Started between $8-72$ hours & $782(61.4)$ & $184(60.9)$ \\
\hline Multidose AZT & $81(6.4)$ & $126(41.7)^{*}$ \\
\hline Single-dose NVP only & $701(55.0)$ & $58(19.3)^{*}$ \\
\hline Did not receive ARVs & $219(17.2)$ & $74(24.6)^{*}$ \\
\hline Unknown & $273(21.4)$ & $44(14.5)^{*}$ \\
\hline
\end{tabular}

${ }^{*} P<.01$ by time period.

${ }^{1}$ Zidovudine, lamivudine, nevirapine highly active antiretroviral therapy.

${ }^{2}$ Zidovudine, lamivudine, ritonavir-boosted lopinavir highly active antiretroviral therapy.

non-HAART regimens for one to 24 weeks was reported in 61 women who received combined zidovudine-lamivudine and 19 who received zidovudine during the pregnancy and caesarean delivery in the period 1999-2008; no multi-dose antiretroviral regimens other than HAART were reported in 2009-2011 (Table 1).

Over $60 \%$ of infants in both periods were delivered by caesarean section and were administered antiretrovirals between 8 and 72 hours after birth; these proportions did not vary significantly by period (Table 1 ). A single dose of nevirapine was administered to most infants who received antiretrovirals from 1999 to 2008, while most infants in the 2009-2011 period who received antiretrovirals received a multidose regimen $(P<.01)$. The proportion who were exclusively formula-fed rose and the proportion who were exclusively breastfed declined significantly.

3.3. Perinatally Acquired HIV Infection. Overall, in both periods combined, HIV infection was diagnosed in 154/1,576 (9.8\%) of perinatally exposed infants and children. The proportion infected declined from $11.1 \%(142 / 1,274)$ in $1999-2008$ to $4.0 \%(12 / 302)$ in $2009-2011(P<.001)$ 
(Table 2). MTCT varied in both periods by whether or not mothers received any antiretrovirals during pregnancy or delivery, and by type of antiretroviral regimen. MTCT rates were similar for infants whose mothers received single-dose nevirapine, regardless of when the nevirapine was given in 1999-2008. The MTCT rate in infants whose mothers received single-dose nevirapine was higher than in those whose mothers received prenatal HAART in 1999-2008 and in $2009-2011$ (6.4\% versus $2.5 \%$ and $5.7 \%$ versus $2.9 \%$, resp.) (Table 2). However, the difference was only statistically significant when the two periods were combined; 9/331 [2.7\%]) infants of mothers who received HAART were infected, versus $35 / 601$ (5.8\%) infants of mothers who received single-dose nevirapine $(P=.03)$.

The MTCT rate among infants of mothers who received HAART varied significantly by how long the mother had received HAART at delivery. HAART duration was shorter (median $=16.5$ days) in mothers whose infants were infected than in mothers whose infants were not infected (median $=161$ days; $P=.003$ ). In the 1999-2008 period, MTCT rates varied considerably by type of delivery, ranging from $6.8 \%$ with cesarean delivery to $23.9 \%$ with vaginal delivery. MTCT rates in the latter period were lower for both types of delivery $(2.5 \%$ and $7.5 \%$ in infants delivered by cesarean and vaginally, resp.). MTCT rates also were higher and tended to vary more by infant-feeding strategy in 1999-2008 than in 2009-2011.

MTCT rates varied from 5.0\% and 6.1\% among infants receiving six weeks of zidovudine or single-dose nevirapine within 72 hours of birth, respectively, to $9.4 \%$ among infants who did not receive any antiretroviral in 1999-2008 (Table 2). In contrast, in 2009-2011, when most infants received antiretrovirals, MTCT rates ranged from 2.4\% to $5.6 \%$ among infants receiving single-dose nevirapine or six weeks of zidovudine, and the differences were not significant. MTCT rates varied significantly in 1999-2008 from $8.1 \%$ in exclusively formula-fed infants to $37.3 \%$ in exclusively breastfed infants. In the latter periods, only 6 infants received breast milk; MTCT rate in those who were exclusively formula fed or receiving unknown nutrition was $4.1 \%$ and $4.0 \%$, significantly lower than in 1999-2008. When the analysis was confined to infants whose mothers had received HAART, were delivered by cesarean and had exclusive formula feeding, the MTCT rate was $1.6 \%$ in 1999 2008 and $2.3 \%$ in 2009-2011. Only 1 of 81 infants born in La Romana in 2011 (1.2\%) was infected.

MTCT rates varied significantly by center (Table 3 ); the MTCT rate in the La Romana center was lower than in other centers in both time periods, but the difference was only significant in 1999-2008 $(P<.001)$. The decline in MTCT risk from 1999-2008 to 2009-2011 was from a higher baseline, of greater magnitude, and statistically significant at centers outside La Romana. The decline in the La Romana center was not statistically significant. When controlled for prenatal antiretroviral use and exclusive formula feeding, no differences in MTCT rates between La Romana and other centers were significant (Tables 2 and 3). Most of the declines in MTCT and the differences by center (Table 3 ) were due to higher use of maternal antiretrovirals and exclusive formula feeding in La Romana in 1999-2008, and higher use of maternal antiretrovirals and exclusive formula feeding in 2009-2011 in all centers.

3.4. Prenatal Screening, Delivery, and Infant Nutrition Practices in the DR. Prenatal care utilization in the DR was estimated at greater than $95 \%$ in 2007 , and varied from $96.6 \%$ in Elias Piña to $99.6 \%$ in Santo Domingo and from $95.1 \%$ in women who had never attended school (uneducated) to $99.6 \%$ among high school graduates [13]. Almost all births $(97.8 \%)$ of births were attended by a health professional, ranging from $84 \%$ in Elias Piña to 99.7\% in Santo Domingo, and from $91.0 \%$ to $99.7 \%$ among uneducated and high school graduates, respectively. The vast majority (97.5\%) of births occurred in hospitals (ranging from $83.3 \%$ to $99.1 \%$ in Elias Piña and Santo Domingo, and from $89.2 \%$ to $98.7 \%$ among uneducated women and high school graduates, resp.). The mean number of prenatal visits was 6.6 . Only $7.8 \%$ of infants aged $0-5$ months were exclusively breastfed [13]. In 2003-2005, in a survey of sentinel sites, over $95 \%$ of women consented to HIV prenatal testing; only $76 \%$ received their test results [21]. In one of the largest DR centers, samples for rapid tests are collected, stored, and performed at the end of the day; the pregnant women were asked to return the next day or week for the results [14]. HIV prevalence in pregnant women varied with the highest prevalence in La Vega (8.4\%), Mao (5.4\%) and La Romana (3.2\%) [16].

Residents of bateyes (generally underserved persons, many of Haitian origin), were surveyed [15]; 92\% of women who had a recent pregnancy had been tested for HIV during the pregnancy, but only 58\% received posttest counseling, including their test results. HIV prevalence among female residents in bateyes was higher $(3.1 \%)$ than in the general female DR population $(0.8 \%)$ and varied from $3.1 \%$ among 20-24 year olds to $4.9 \%$ among $30-34$ year olds, and was highest (7.9\%) among women aged 45-49 years old. HIV prevalence in DR women varied by educational attainment, from less than $.3 \%$ in high school graduates to $3.7 \%$ in uneducated women $[12,16]$. HIV prevalence in the general population varied geographically, with highest prevalence in the northernmost border with Haiti $(1.6 \%)$ and the eastern sugar-growing region, where La Romana and most bateyes are located $(1.2 \%)$, and the lowest prevalence in the middleborder region $(.4 \%)$.

\section{Discussion}

The data in this study suggest that elimination of MTCT in the DR is feasible and attainable. Encouraging signs include the significant increases in proportion of women who received antiretrovirals during their pregnancy, with most of that increase being in HAART use in women without severe immunodeficiency, and the proportion of infants receiving exclusive formula feeding. These increases coincide with and largely explain the decrease in MTCT observed in the years since the publication of guidelines for use of HAART for pMTCT. They also coincide with some national 
TABLE 2: HIV mother-to-child transmission risk in the 1999-2008 and 2009-2011 periods, by prenatal antiretroviral, delivery route, and infant feeding prevention strategies, Dominican Republic.

\begin{tabular}{|c|c|c|}
\hline All perinatally exposed & $142 / 1,274(11.1 \%)$ & $12 / 302(4.0 \%)^{*}$ \\
\hline Any maternal antiretrovirals & $54 / 861(6.3)$ & $10 / 267(3.7)$ \\
\hline HAART $^{1}$ all & $4 / 157(2.5)$ & $5 / 174(2.9)$ \\
\hline $\mathrm{AZT}+3 \mathrm{TC}+\mathrm{NVP}^{2}$ & $4 / 157(2.5)$ & $0 / 59$ \\
\hline $\mathrm{AZT}+3 \mathrm{TC}+$ Lopinavir $/ \mathrm{r}^{3}$ & - & $5 / 115(4.3)$ \\
\hline Non-HAART multidose & \multirow{2}{*}{$4 / 80(5.0)$} & \multirow{2}{*}{ - } \\
\hline AZT-containing regimen & & \\
\hline Single-dose NVP ALL & $32 / 506(6.3)$ & $3 / 60(5.0)$ \\
\hline 8 hours precaesarean & $25 / 395(6.3)$ & $0 / 2$ \\
\hline Labor onset & $5 / 78(6.4)$ & - \\
\hline At delivery & $2 / 30(6.7)$ & - \\
\hline Unspecified & - & $3 / 58(5.2)$ \\
\hline Unknown & $14 / 211(11.6)$ & $2 / 30(6.7)$ \\
\hline None & $88 / 413(21.3)^{\dagger}$ & $2 / 35(5.7)^{*}$ \\
\hline \multicolumn{3}{|l|}{ Delivery } \\
\hline Vaginal & $68 / 285(23.9)^{\dagger}$ & $6 / 80(7.5)^{*}$ \\
\hline Caesarean & $60 / 882(6.8)$ & $5 / 199(2.5)^{*}$ \\
\hline Unknown & $14 / 107(13.1)$ & $1 / 23(4.3)^{*}$ \\
\hline \multicolumn{3}{|l|}{ Infant antiretrovirals } \\
\hline Single-dose Nevirapine (SD NVP) & $48 / 782(6.1)$ & $1 / 41(2.4)$ \\
\hline SD-NVP after 72 hours & $1 / 13(7.6)$ & - \\
\hline Zidovudine for six weeks & $7 / 139(5.0)$ & $7 / 126(5.6)$ \\
\hline Unknown/other/none & $32 / 340(9.4)^{\dagger}$ & $4 / 135(3.0)$ \\
\hline \multicolumn{3}{|l|}{ Infant feeding } \\
\hline Breast fed only & $25 / 67(37.3)$ & $0 / 2$ \\
\hline Mixed & $16 / 43(37.2)$ & $0 / 4$ \\
\hline Formula only & $79 / 972(8.1)^{\dagger}$ & $11 / 271(4.1)^{*}$ \\
\hline Unknown & $22 / 192(11.5)$ & $1 / 25(4.0)^{*}$ \\
\hline $\begin{array}{l}{ }^{*} P<.05 \text {, comparison by time period, } 1999- \\
{ }^{\dagger} P<.01 \text { within period, by strategy. } \\
{ }^{1} \text { Highly active antiretroviral therapy. } \\
{ }^{2} \text { AZT-3TC-NVP: zidovudine, lamivudine, } \mathrm{n} \\
{ }^{3} \text { AZT-3TC-Lop/r: zidovudine, lamivudine, } 1\end{array}$ & & \\
\hline
\end{tabular}

trends that suggest a possible decline in HIV prevalence and unsafe sex practices, and documented increase in HAART use nationwide $[1,12,16,22,23]$.

These encouraging findings contrast with persistent problems implementing the fundamentals of effective pMTCT, particularly informing pregnant women of their HIV status, and linking them to care and HAART. It is clear that all of the components of the "cascade" needed for MTCT elimination are present in the DR. These include high use of prenatal care and institutional delivery by trained personnel; high acceptability of prenatal HIV testing, formula feeding, and prenatal, perinatal, and infant antiretroviral use, and legislation that offers some protection, though modest and with draconian components, for people living with HIV $[24,25]$.

Yet the "weak links" in this system are unfortunately at points where they threaten the many strengths of the program. The principal weak links include the high proportion of women tested in prenatal care who do not receive their results. According to national guidelines, prenatal HIV testing in the DR is to be performed using rapid antibody tests; results of samples that test positive are then confirmed with another rapid test that uses a different principle, according to WHO guidelines [26], allowing infected pregnant women to receive at least presumptive, if not confirmed, positive test results in the same visit in which the sample for testing is obtained. Yet according to an observational study and a case report in some centers, samples were stored as they were collected and rapid tests run once a day; women were asked to return for their results, and posttest counseling sometimes consisted of reporting that the test "needs to be repeated" without clarifying that it was a presumptive positive test, leading to women delivering without an HIV diagnosis, MTCT, and maternal and infant HIV progression and death $[14,27,28]$.

Another potential weakness in the cascade is the linkage to specialized care for an expedited CD4+ T-lymphocyte count to determine which HAART and other treatments are 
TABLE 3: Mother to child transmission (MTCT) rates by period (before recommendation of highly active antiretroviral therapy (HAART) for prevention of MTCT (1999-2008), and after (2009-2011) by center (La Romana center versus others) and prevention strategies, Dominican Republic.

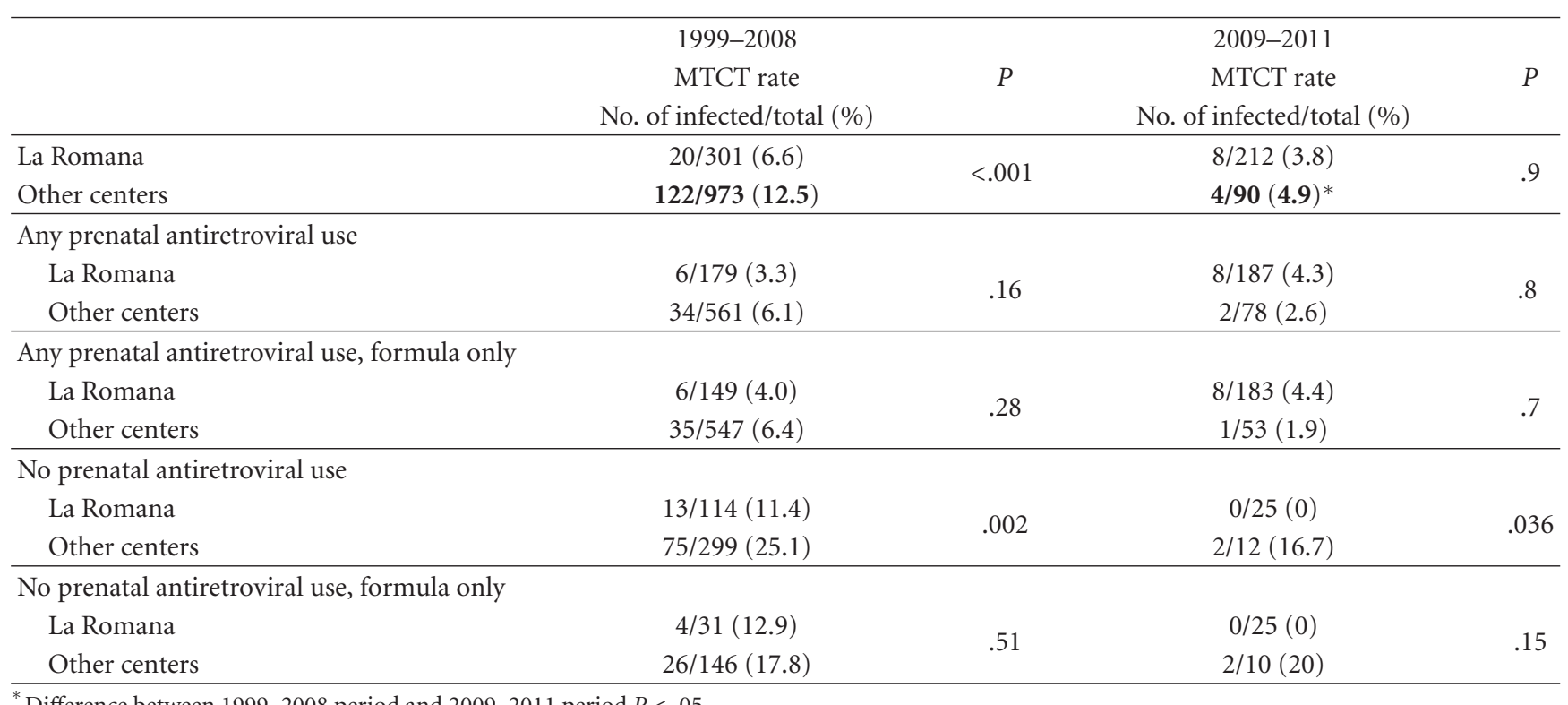

${ }^{*}$ Difference between 1999-2008 period and 2009-2011 period $P<.05$.

indicated for the mother's health and pMTCT. In at least one center, in La Romana, this is facilitated by having the counselor (usually a woman living with HIV herself [21]), contact an HIV comprehensive care unit to have staff from that unit personally meet with and expedite the pregnant woman's linkage to care. Models of this type in the DR have demonstrated the feasibility of effective linkage to care for pMTCT and maternal health. However, in centers whose referral process consists of asking the women to schedule an appointment with a specialized clinic, many women will not receive HAART, even if they continue prenatal care. Moreover, despite a high level of utilization of prenatal care and hospital delivery, the populations least likely to access these services include the most HIV-affected [12, 15]; alternative methods of reaching these populations are essential.

The dependence on ritonavir-boosted lopinavir (instead of efavirenz, as recommended by the WHO [29]) for HAART for women who are not severely immunesuppressed because of probably exaggerated concerns about its teratogenicity may limit their antiretroviral options in the future, and incurs a prohibitive cost compared to efavirenz [6]. Finally, although there is growing acceptance of the rights of people living with HIV, stigma, and discrimination continue to affect women with HIV in the DR $[30,31]$. These women may also be unaware of their right to HAART at no cost $[24,25]$. In the past, some HIV-infected women who did not receive their results found that they were given instead to employers, landlords or medical personnel; they were denied employment, housing, and elective medical services, including cesarean delivery for pMTCT $[30,31]$. The new law criminalizes even protected sex with an uninfected partner; thus, some DR women who know or suspect their status may consider the risks of testing to be unacceptably high [24].

This study has several strengths. It contains data on by far the largest number of HIV perinatally exposed infants of any study conducted in the DR. A considerable amount of data was collected on infants from a large number of diverse centers distributed widely throughout the country. However, this analysis has several problems that limit its generalizability in characterizing MTCT in the DR. First, surveillance for perinatal HIV exposure that depends on testing living infants and children is susceptible to the important survival bias related to the high mortality (up to $30 \%$ ) of untreated perinatally acquired HIV infection in the first two years of life [28]. Also, these data do not represent the majority of cases of perinatal exposure in the DR; the estimates of HIV prevalence in some DR pregnant and childbearing populations, and the numbers of HIV-infected women delivering each year (notably in La Romana and some sentinel surveillance hospitals) have been confirmed by several studies [13, 17], enabling reliable estimates of the proportion of perinatally exposed infants that are returned for virologic testing. At the national level, the estimated number of women with HIV who deliver annually (at least 1,400 to over 5,700) is much higher than the number of infants tested. This study represents at best a small proportion of DR perinatally exposed infants $[6,13$, $16,17,21]$, and greatly over represents the centers (the NGO in La Romana and one of the public Maternity Hospitals in Santo Domingo) [14] with the best outcomes.

Despite these limitations, this analysis represents the first glimpse of the impact of the historic recommendations for HAART use for pMTCT with a large, representative 
database. It suggests that MTCT elimination is possible with current tools; indeed, some of the DR's 2012 benchmarks were achieved in 2011 [16]. Barriers to elimination are related to failures to ensure that HIV-infected women are informed of their status and linked to care. Structural barriers limit healthcare access for the DR's most HIVaffected, most marginalized populations. Surmounting these barriers requires innovative strategies to close the gaps in this promising model [32].

\section{Acknowledgments}

The authors gratefully acknowledge the contributions of José Ledesma, M.D., Ana Gloria Bido, M.D., and David Martin, M.D., of the Clinton Foundation HIV/AIDS Initiative, Santo Domingo, who supported all aspects of the use of dried blood spots for collection of specimens for virologic testing, in collaboration with the Dirección General de Control de Infecciones de Transmisión Sexual y SIDA; Susan A. Fiscus, Ph.D., Mark Turner, and the staff at the University of North Carolina Retrovirology Laboratory, Chapel Hill, North Carolina; the Virology Laboratory of the National Institute of Communicable Diseases in Johannesburg, South Africa, and the National Virology Laboratory for performing the virologic testing; the staff of the 20 participating HIV MTCT programs, including counselors, physicians, nursing, and laboratory staff; the mothers and guardians of the infants who consented to virologic testing and the peer-communitybased home visitors and caregivers who promoted adherence to prenatal and infant antiretroviral and safe artificial formula use.

\section{References}

[1] Joint United Nations Programme on HIV/AIDS, UNAIDS World AIDS Day Report, http://www.unaids.org/en/media/ unaids/contentassets/documents/unaidspublication/2011/JC2216_WorldAIDSday_report_2011_en.pdf, 2011.

[2] World Health Organization, "Progress report 2011: global HIV/AIDS response,” Tech. Rep., WHO, Geneva, Switzerland, 2011.

[3] S. K. Whitmore, A. W. Taylor, L. Espinoza, R. L. Shouse, M. A. Lampe, and S. Nesheim, "Correlates of mother-to-child transmission of HIV in the United States and Puerto Rico," Pediatrics, vol. 129, no. 1, pp. e74-e81, 2012.

[4] C. L. Townsend, M. Cortina-Borja, C. S. Peckham, A. De Ruiter, H. Lyall, and P. A. Tookey, "Low rates of motherto-child transmission of HIV following effective pregnancy interventions in the United Kingdom and Ireland, 2000-2006," AIDS, vol. 22, no. 8, pp. 973-981, 2008.

[5] S. Tariq, C. L. Townsend, M. Cortina-Borja et al., "Use of zidovudine-sparing HAART in pregnant HIV-infected women in Europe: 2000-2009," Journal of Acquired Immune Deficiency Syndromes, vol. 57, no. 4, pp. 326-333, 2011.

[6] N. C. Schmidt, J. Roman-Pouriet, A. D. Fernandez, C. M. Beck-Sagué, J. Leonardo-Guerrero, and S. W. Nicholas, "Costs and benefits of multidrug, multidose antiretroviral therapy for prevention of mother-to-child transmission of HIV in the Dominican Republic," International Journal of Gynecology \& Obstetrics, vol. 116, no. 3, pp. 219-222, 2012.
[7] B. Robberstad and B. Evjen-Olsen, "Preventing mother to child transmission of HIV with highly active antiretroviral treatment in Tanzania-a prospective cost-effectiveness study," Journal of Acquired Immune Deficiency Syndromes, vol. 55, no. 3, pp. 397-403, 2010.

[8] S. Orlando, M. C. Marazzi, S. Mancinelli et al., "Costeffectiveness of using HAART in prevention of mother-tochild transmission in the DREAM-project Malawi," Journal of Acquired Immune Deficiency Syndromes, vol. 55, no. 5, pp. 631$634,2010$.

[9] Joint United Nations Program on HIV/AIDS (UNAIDS) and World Health Organization (WHO), AIDS Epidemic Update 2009, World Health Organization, Geneva, Switzerland, 2009.

[10] United States Agency for International Development, HIV/ AIDS Health profile for the Caribbean Region, http://transition.usaid.gov/our_work/global_health/aids/Countries/lac/caribbean_profile.pdf, Pages 1-6, 2012.

[11] Centers for Disease Control and Prevention, "Revised surveillance case definitions for HIV infection among adults, adolescents, and children aged $<18$ months and for HIV infection and AIDS among children aged 18 months to $<13$ yearsUnited States, 2008," Morbidity and Mortality Weekly Report, vol. 57, no. 10, pp. 5-8, 2008.

[12] Centro de Estudios Sociales and Demográficos (CESDEM) y Macro International Inc, Encuesta Demográfica y de Salud 2007. (Demographic and Health Survey, 2007, CESDEM y Macro International Inc., Santo Domingo, República Dominicana, 2008.

[13] J. Román-Poueriet, A. D. Fernandez, C. M. Beck-Sagué et al., "HIV infection and prevention of mother-to-child transmission in childbearing women: La Romana, Dominican Republic, 2002-2006," Revista Panamericana de Salud Publica/Pan American Journal of Public Health, vol. 26, no. 4, pp. 315-323, 2009.

[14] M. Connolly, C. Bautista-Soriano, and A. Castro, "Utilización formulario de la Historia Clínica Perinatal y Neonatal CLAP/OPS-OMS. (Use of the Clinical Perinatal and Neonatal History Forms CLAP/PAHO-WHO) Publicación Bol.," CENISMI, vol. 19, no. 3, pp. 17-23, 2009.

[15] CESDEM and Macro International Inc., Encuesta Socioemográfica y sobre VIH/SIDA en los Bateyes Estatales de la República Dominicana 2007, CESDEM y Macro International Inc., Santo Domingo, Dominican Republic, 2008.

[16] Consejo Presidencial del SIDA (COPRESIDA), "Ministerio de Salud Pública y Asistencia Social Informe de estimaciones y proyecciones de prevalencia de carga de enfermedad año 2009," http://copresida.gob.do/.../Informe_Estimaciones_2009, 2011.

[17] E. Perez-Then, R. Peña, M. Tavarez-Rojas et al., "Preventing mother-to-child HIV transmission in a developing country: the Dominican Republic experience," Journal of Acquired Immune Deficiency Syndromes, vol. 34, no. 5, pp. 506-511, 2003.

[18] K. V. Shepard, "Clarification of risk factors for severe, life-threatening and fatal hepatotoxicity with VIRAMUNE (nevirapine)," 2004, http://www.fda.gov/Safety/MedWatch/ SafetyInformation/SafetyAlertsforHumanMedicalProducts/ ucm166533.htm?utm_campaign=Google2\&utm_source=fdaSearch\&utm_medium $=$ website\&utm_term $=c d 4$ nevirapine\& utm_content $=1$ \&utm_source $=$ fdaSearch\&utm_medium $=$ website\&utm_term=cd4nevirapine\&utm_content=1, 2012.

[19] G. G. Sherman, G. Stevens, S. A. Jones, P. Horsfield, and W. S. Stevens, "Dried blood spots improve access to HIV diagnosis 
and care for infants in low-resource settings," Journal of Acquired Immune Deficiency Syndromes, vol. 38, no. 5, pp. 615-617, 2005.

[20] A. G. Dean, K. M. Sullivan, and M. M. Soe, "Epi Info and OpenEpi in epidemiology and clinical medicine. Health applications of free software," http://www.epiinformatics.com/, 2012.

[21] E. Perez-Then, C. Bautista Soriano, R. Espinal et al., Centro Nacional de Investigaciones de Salud Materno-Infantil (CENISMI). Consolidation of strategies for reduction of HIV MTCT in the Dominican Republic (CENISMI-Glaser Project). CENISMI, Santo Domingo, DR.

[22] P. Rojas, R. Malow, B. Ruffin, E. M. Rothe, and R. Rosenberg, "The HIV/AIDS epidemic in the Dominican Republic: key contributing factors," Journal of the International Association of Physicians in AIDS Care, vol. 10, no. 5, pp. 306-315, 2011.

[23] D. T. Halperin, E. A. De Moya, E. Pérez-Then, G. Pappas, and J. M. Garcia Calleja, "Understanding the HIV epidemic in the Dominican Republic: a prevention success story in the Caribbean?" Journal of Acquired Immune Deficiency Syndromes, vol. 51, no. 1, pp. S52-S59, 2009.

[24] L. Fernandez, Ley 135-11 VIH/SIDA, Promulgated by the President, Santo Domingo, Dominican Republic, 2011.

[25] F. A. Cuello Camilo, "Statement by H. E. Ambassador Frederico Alberto Cuello CamiloRepresentative of the Dominican Republic to the United Nations," in Proceedings of the High Level Meeting of VIH/SIDA, 2012, http://www.un.org/en/ga/ aidsmeeting2011/pdf/dominican\%20rep.pdf.

[26] World Health Organization, Rapid HIV Tests. 2004. Guidelines for Use in HIV Testing and Counselling Services in ResourceConstrained Settings, WHO, Geneva, Switzerland.

[27] A. Koppel, J. Leonardo-Guerrero, S. Rives, N. Paniagua-Torres, C. Sparrow, and C. M. Beck-Sagué, "Immune reconstitution inflammatory syndrome due to Mycobacterium bovis bacillus Calmette-Guérin in infants receiving highly active antiretroviral therapy: a call for universal perinatal rapid HIV testing prior to administration of BCG immunization of neonates," Journal of Tropical Pediatrics, vol. 56, no. 4, Article ID fmp115, pp. 280-283, 2009.

[28] M. J. Mayaux, M. Burgard, J. P. Teglas et al., "Neonatal characteristics in rapidly progressive perinatally acquired HIV-1 disease," Journal of the American Medical Association, vol. 275, no. 8, pp. 606-610, 1996.

[29] World Health Organization, "Antiretroviral drugs for treating pregnant women and preventing HIV infection in infants: recommendations for public health approaches," http://www .who.int/hiv/pub/guidelines/pmtctguidelines3.pdf, 2012.

[30] Cáceres Ureña FI. República Dominicana. Estigma y discriminación en personas que viven con el VIH, http://www.stigmaindex.org/download.php?id=44, 2012.

[31] M. Møllmann, A Test of Inequality: Discrimination against women living with HIV in the Dominican Republic. Human Rights Watch, http://www.hrw.org/sites/default/files/reports/ dr0704.pdf, 16, 2004.

[32] S. W. Nicholas, "Towards the elimination of pediatric HIV/ AIDS in New York State," Journal of Public Health Management and Practice, vol. 16, no. 6, pp. 509-511, 2010. 


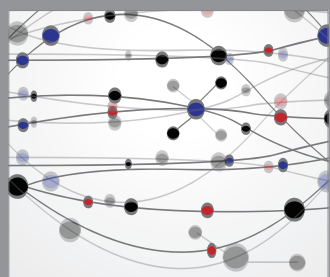

The Scientific World Journal
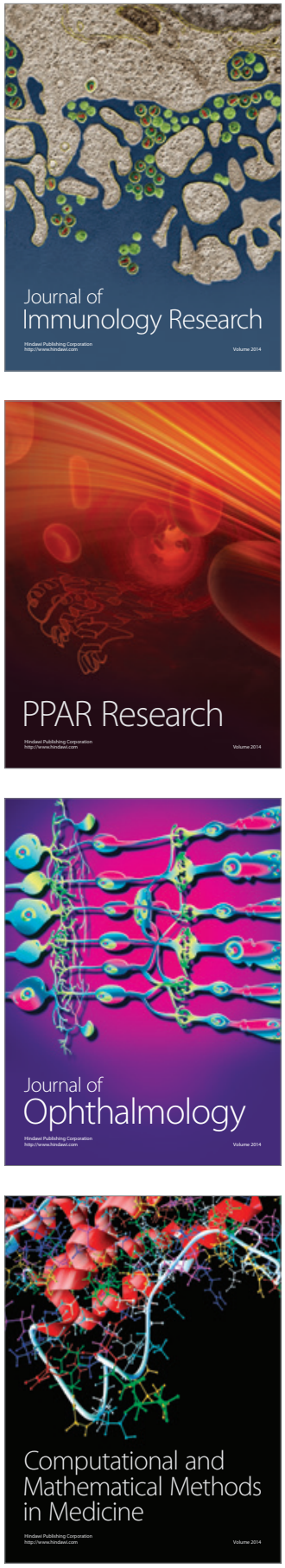

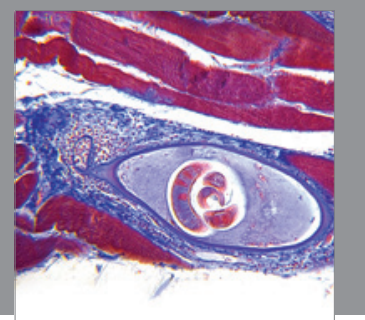

Gastroenterology

Research and Practice
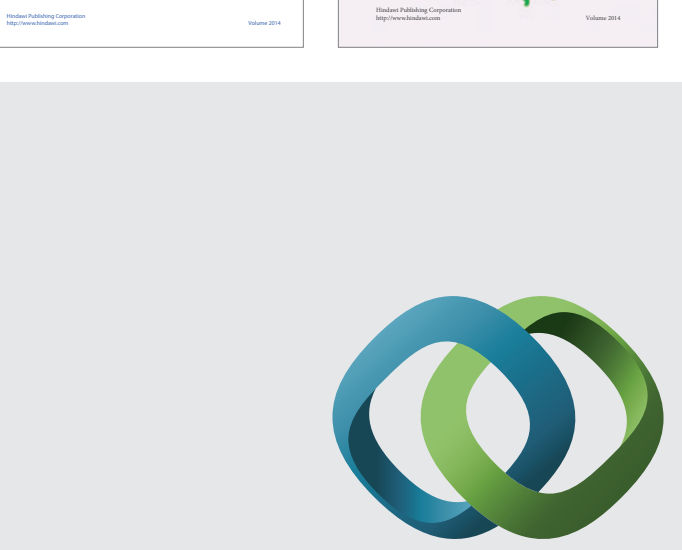

\section{Hindawi}

Submit your manuscripts at

http://www.hindawi.com
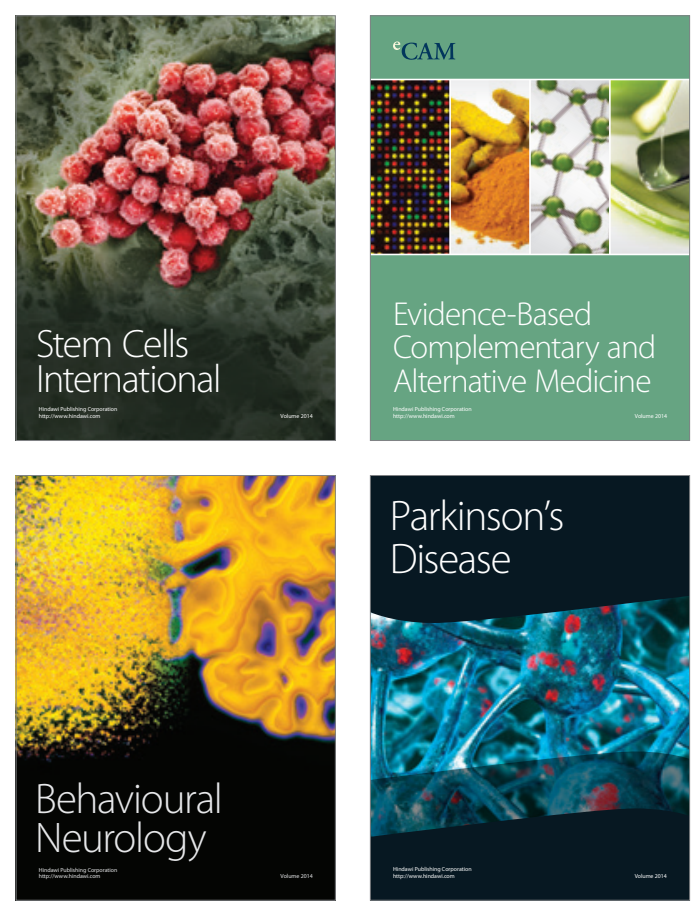

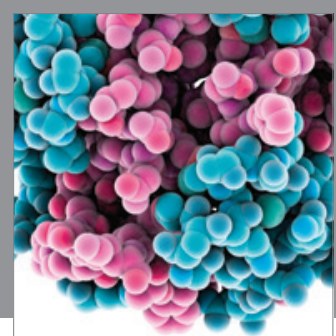

Journal of
Diabetes Research

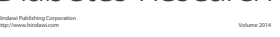

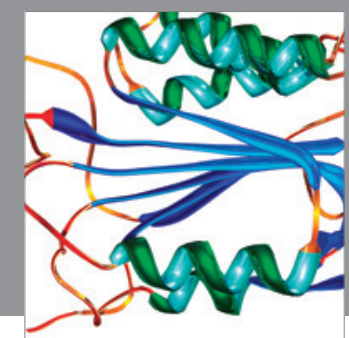

Disease Markers
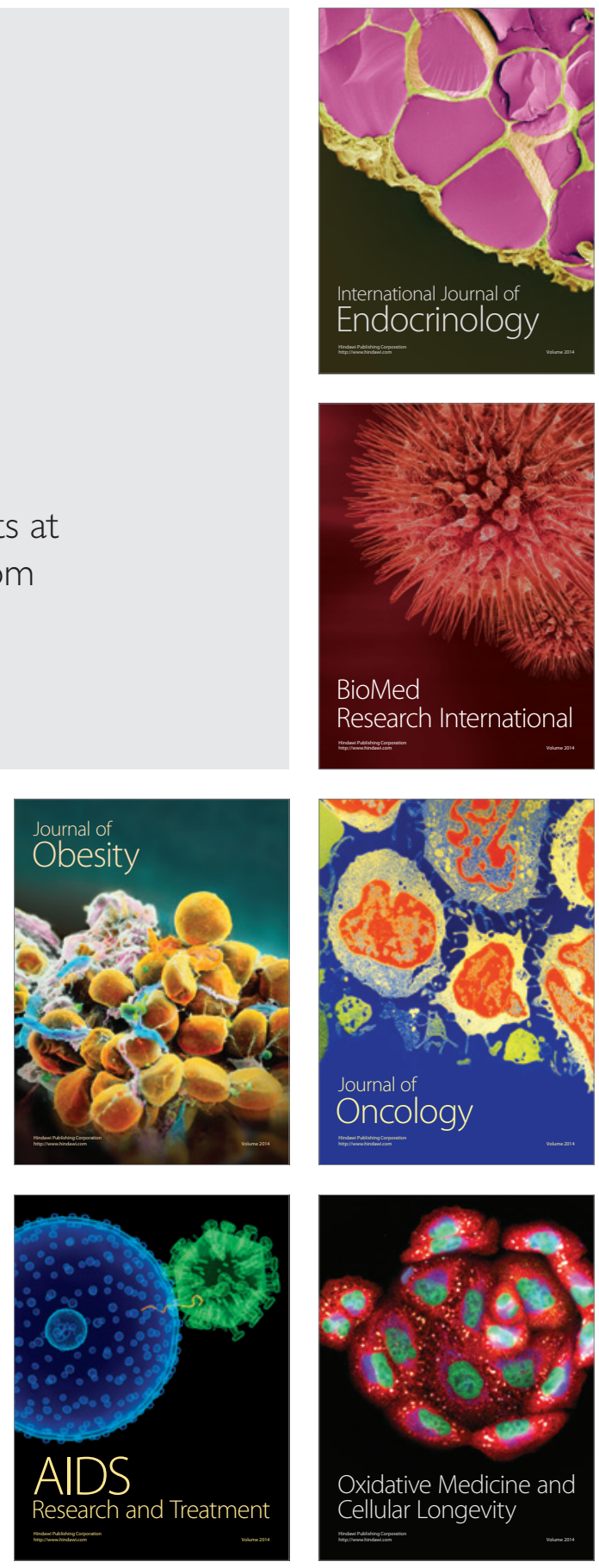